\title{
Practical applications of sperm selection techniques for improving reproductive efficiency
}

\author{
J.M. Morrell ${ }^{1}$, M. Sabes Alsina, M.C. Abraham, Y. Sjunnesson
}

Division of Reproduction, Clinical Sciences, Swedish University of Agricultural Sciences, Uppsala, Sweden.

\begin{abstract}
Several selection techniques are available for processing spermatozoa. Apart from sperm washing to remove seminal plasma, only "swim-up" and colloid centrifugation have been used to any extent to prepare spermatozoa for in vitro fertilization, and only colloid centrifugation has been used to prepare sperm samples for artificial insemination. Single-layer centrifugation (SLC) through a species-specific colloid has been shown to be effective in selecting spermatozoa with good motility, normal morphology and intact chromatin in a range of species. This method is less timeconsuming than swim-up, and has been scaled-up to allow whole ejaculates to be processed in a practical manner. The applications of SLC are as follows: to improve sperm quality in insemination doses or in samples for in vitro fertilization, to increase the shelf life of normal sperm doses, to remove pathogens (viruses, bacteria), to improve cryosurvival by removing dead and dying spermatozoa before freezing or after thawing, to select spermatozoa for intracytoplasmic sperm injection, and to aid conservation breeding.
\end{abstract}

Keyword: artificial insemination, chromatin integrity, filtration, in vitro fertilization, migration.

\section{Introduction}

The purpose of this review is to compare and contrast the sperm selection techniques that are available for selecting spermatozoa for use in various Assisted Reproduction Technologies (ART), with particular reference to those techniques used in preparing sperm samples for artificial insemination (AI) and in vitro fertilization (IVF) or intracytoplasmic sperm injection (ICSI). The reasons for needing to select sperm will be described first, followed by a summary of the techniques available. The main part of the review will focus on colloid centrifugation, particularly Single Layer Centrifugation as an emerging (and promising) technology for sperm selection.

\section{Why are sperm selection techniques needed?}

It is difficult to produce good quality blastocysts from poor quality gametes. Semen samples contain a heterogeneous population of spermatozoa, some of which may possess the attributes necessary for successful fertilization. The "desirable" spermatozoa are thought to be selected by various mechanisms within the female reproductive tract, with the result that the small number of spermatozoa found in the vicinity of the oocyte are typically those best able to penetrate the zona pellucida and fertilize the oocyte. When using ART, however, these natural selection mechanisms are circumvented, allowing most spermatozoa to be found near the oocyte. The main reason for using a sperm selection technique in assisted reproduction is to select good quality (hopefully, functional) spermatozoa and to separate them from the rest of the sample, including any seminal plasma or extender that may be present (Morrell and Rodriguez-Martinez, 2009). Since seminal plasma contains decapacitation factors, spermatozoa must be removed from seminal plasma before they can be used in IVF. In the female reproductive tract, separation of spermatozoa from seminal plasma and selection of normal spermatozoa occurs at various sites, ranging from mechanical separation in the cervix (in animals that have vaginal deposition of semen during mating) to selective binding to oviductal epithelial cells (Suarez, 2007). The separation techniques that are performed in the laboratory mimic these selection mechanisms and are therefore biomimetic techniques.

Spermatozoa face many challenges in the female reproductive tract before they can reach the site of fertilization and penetrate the oocyte. There are fewer challenges to overcome in the in vitro situation, for example, spermatozoa do not have to navigate their way through the reproductive tract to reach the oocyte, and therefore the parameters of sperm quality linked to fertilizing ability, and the timing of events such as capacitation, may be different in vitro and in vivo. It is not only penetration and activation of the oocyte that is important but also the ability of the zygote to continue to develop; sperm quality, particularly chromatin integrity, appears to be important in this process.

\section{Which sperm selection techniques are available and which are used in practice?}

In previous reviews, sperm preparation techniques have been divided into those that separate spermatozoa from seminal plasma e.g. sperm washing and simple filtration, and those that also select a subpopulation of spermatozoa, e.g. migration and colloid centrifugation. These techniques have been described in detail previously (Morrell and Rodriguez-Martinez, 2009, 2010; Morrell, 2012) and are summarized in Table 1. The methods that have been used consistently in practice are sperm migration (in the form of "swimup") and colloid centrifugation. Both of these techniques separate the spermatozoa from seminal plasma and extender but whereas "swim-up" separates motile spermatozoa from immotile ones, colloid centrifugation allows the separation of morphologically normal, motile spermatozoa with intact chromatin from the rest of the sample (Morrell et al., 2009), and also 
removes seminal plasma proteins that are coating the surface of the spermatozoa (Kruse et al., 2011). Swimup has been used to prepare spermatozoa for IVF, whereas colloid centrifugation has been used for both IVF and AI. The disadvantages of swim-up are that it takes approximately 45-60 $\mathrm{min}$ to do and only 10-20\% of the spermatozoa in the sample are recovered. For colloid centrifugation, only 25 min preparation time is needed (including the centrifugation) and a recovery rate of $>50 \%$ is commonly achieved (Thys et al., 2009), although this does depend on the sperm quality of the original sample.

The remainder of this review will focus on colloid centrifugation for preparation of sperm samples for ART.

Table 1. Sperm separation and selection methods.

\begin{tabular}{|c|c|c|c|c|}
\hline Method & $\begin{array}{l}\text { Seminal plasma } \\
\text { removed }\end{array}$ & Sperm quality enhanced & $\begin{array}{l}\text { Removal of potential } \\
\text { pathogens }\end{array}$ & $\begin{array}{l}\text { Fertility } \\
\text { improved }\end{array}$ \\
\hline $\begin{array}{l}\text { Fractionation during } \\
\text { collection }\end{array}$ & Mostly & $\begin{array}{l}\text { Survival during storage } \\
\text { increased }\end{array}$ & $\begin{array}{l}\text { May be decreased } \\
\text { Bacterial contamination } \\
\text { may be reduced }\end{array}$ & Possibly \\
\hline $\begin{array}{l}\text { Sperm washing; simple } \\
\text { filtration }\end{array}$ & yes & $\begin{array}{l}\text { Kinematics may change; } \\
\text { survival during storage } \\
\text { increased }\end{array}$ & No & No \\
\hline Sperm migration & Yes & Selection for motility & $\begin{array}{l}\text { Viruses removed, bacteria } \\
\text { may be present }\end{array}$ & Possibly \\
\hline $\begin{array}{l}\text { Filtration eg through } \\
\text { glass wool, Sephadex }\end{array}$ & Partial removal & $\begin{array}{l}\text { Selection for morphological } \\
\text { normality, acrosome integrity }\end{array}$ & No & Possibly \\
\hline Colloid centrifugation & Yes & $\begin{array}{l}\text { Selection for motile, } \\
\text { morphologically normal, intact } \\
\text { membrane and acrosome, intact, } \\
\text { chromatin. }\end{array}$ & Mostly & Yes \\
\hline Hyaluronic acid binding & $\begin{array}{l}\text { (spermatozoa } \\
\text { are washed first) }\end{array}$ & $\begin{array}{l}\text { Selection for binding to HA } \\
\text { droplets or hyaluronan }\end{array}$ & Not tested & Yes \\
\hline $\begin{array}{l}\text { Sexing by flow } \\
\text { cytometry }\end{array}$ & Yes & $\begin{array}{l}\text { No, selection is for } \mathrm{X} \text { or } \mathrm{Y} \\
\text { chromosome }\end{array}$ & Not reported & No \\
\hline
\end{tabular}

Modified from Morrell and Rodriguez-Martinez (2009).

\section{Background to colloid centrifugation}

Heterogeneous cell populations can be separated into sub-populations according to density by centrifugation through colloids, a technique commonly known as density gradient centrifugation. During centrifugation on a gradient, cells move to a point corresponding to their own density, known as the isopycnic point (Pertoft, 2000). The apparent densities of spermatozoa from several species were identified by Oshio (1988). However, when colloids are used to prepare spermatozoa for ART, the density of the bottom layer of colloid is chosen to be less than that of mature spermatozoa, with the result that the spermatozoa pass through the colloid and collect in a pellet at the bottom of the tube. Immotile, morphologically abnormal, acrosome reacted spermatozoa or those with damaged chromatin are mostly retained at the interface between the semen and the colloid (Morrell et al., 2009).

The first colloid to be used for sperm preparation consisted of polyvinylpyrrolidone (PVP)coated silica particles (Percoll, GE, Uppsala, Sweden) e.g. Serafini et al. (1990). Different densities of Percoll were produced by mixing the Percoll with various salt solutions, for example, TALP (Parrish et al., 1995) concentrated Tyrodes salts (Matás et al., 2010). However, approximately 20 years ago there was some controversy about whether the PVP-coating could be toxic to spermatozoa (Avery and Greve, 1995). Although Motoshi et al. (1996) observed no detrimental effects to bull spermatozoa after their exposure to PVP when capturing them for ICSI, there were reports of toxicity to mouse spermatozoa similarly exposed
(Mizuno et al., 2002). This issue has not been satisfactorily resolved, partly because of species differences but also differences in PVP from various sources (Balaban et al., 2003). Subsequently it became apparent that some batches of Percoll had high endotoxin levels that were detrimental to sperm survival, which necessitated testing each batch of Percoll for sperm toxicity prior to use (Mortimer et al., 2000). The subsequent availability of silane-coated silica colloids provided an alternative to Percoll and resolved both the toxicity and the endotoxin issues.

Other issues with Percoll were related to loss of the acrosome during passage through the colloid. Cesari et al. (2006) reported a significant proportion of lost acrosomes from bull spermatozoa after preparation by Percoll density centrifugation compared to swim-up. This observation was in contrast to Somfai et al. (2002) who observed that preparation by Percoll density gradient resulted in a higher proportion of spermatozoa with intact acrosomes than swim-up. However, the centrifugation force used was greater in the study by Cesari et al. (700 g compared to $300 \mathrm{~g}$ ) which may have contributed to the lost acrosomes.

A variant of colloid centrifugation using only one layer of colloid (in which case there is no gradient) has been developed. This method, known as Single Layer Centrifugation (SLC), has gained popularity when preparing spermatozoa for AI since larger volumes of semen can be processed in this manner than by density gradient. It is too time-consuming and impractical to attempt to layer several colloids of different densities in large tubes to create density gradients for large volumes of semen. Initially the SLC 
technique was developed in $15 \mathrm{ml}$ centrifuge tubes using $4 \mathrm{ml}$ colloid but is was subsequently scaled-up to use $15 \mathrm{ml}$ colloid in a $50 \mathrm{ml}$ tube (Morrell et al., 2009), then to $20 \mathrm{ml}$ colloid in a $100 \mathrm{ml}$ tube and eventually to $150 \mathrm{ml}$ colloid in a $500 \mathrm{ml}$ tube (Morrell et al., 2011). Subsequently a small volume of colloid $(1 \mathrm{ml})$ was used to prepare thawed red deer semen in an eppendorf tube (Anel-López et al., 2015). Recently a comparison was made between the original $4 \mathrm{ml}$ colloid (Small SLC) and $1 \mathrm{ml}$ of colloid, either in a $15 \mathrm{ml}$ centrifuge tube (Mini-SLC) or in an eppendorf tube (Mini-EP) when preparing bull spermatozoa for IVF; although the sperm quality was good in all three variants, the sperm yield was highest in the Mini-SLC. Fortyfour blastocysts were produced from the Mini-SLC sperm preparation compared to 36 from the control (swim-up) preparation (Abraham et al., 2016). Although the difference was not statistically significant for this particular bull, the spermatozoa from this bull are known to work well in IVF (Y Sjunnesson; 2016; Division of Reproduction, Clinical Sciences, Swedish University of Agricultural Sciences, Uppsala, Sweden; personal communication). It would be interesting to try the Mini-SLC preparation technique for semen that does not normally work well in IVF.

\section{Properties of SLC-selected sperm samples}

The SLC technique has been used in a variety of species to prepare sperm samples for assisted reproduction. Most of the work on SLC has been carried out with stallion semen, mainly to prepare spermatozoa for AI but also for cryopreservation and for ICSI (Colleoni et al., 2011). The SLC-selected sperm samples tend to have better sperm quality than unselected controls, in terms of sperm motility (kinematics measured by computer assisted sperm analysis), membrane integrity, chromatin integrity, and normal morphology. Selected stallion sperm samples survive longer than unselected samples (Johannisson et al., 2009; Morrell et al., 2010) and retain their fertilizing capacity during cold storage for at least $96 \mathrm{~h}$ (Lindahl et al., 2012). They show improved pregnancy rates compared to controls, even for stallions of "normal" fertility (Morrell et al., 2014b). Spermatozoa can be separated from most of the bacteria contaminating a stallion ejaculate and also from equine arteritis virus in the semen of "shedding" stallions (Morrell et al., 2103). Selected spermatozoa show improved cryosurvival compared to unselected controls (Hoogewijs et al., 2011), and survive longer postthawing (Hoogewijs et al., 2012). An additional interesting observation is that the yield of stallion spermatozoa after SLC was highly correlated with the fertility of the male after insemination of unselected samples (Morrell et al., 2014d), implying that SLC could be used as a diagnostic tool to indicate the potential fertility of a breeding stallion.

Boar sperm samples may have very good quality initially, as assessed by commonly used laboratory assays, which makes it difficult to see an improvement after selection. However, some improvement in sperm quality after SLC has been reported (Morrell et al., 2009). The SLC-selected samples showed enhanced ability to fertilize oocytes in IVF, necessitating a reduction in sperm dose to avoid polyspermy (Sjunnesson et al., 2013). The selected samples survive cryopreservation better than unselected samples (Martinez-Alborcia et al., 2012). Boar spermatozoa can be separated from bacteria contaminating the ejaculate during semen collection (Morrell and Wallgren 2011) and from porcine circovirus added to the ejaculate (Blomquist et al., 2011).

Most of the studies with bull semen have used SLC to prepare spermatozoa for IVF e.g. Thys et al. (2009), but there are some reports of its use with fresh semen e.g. Goodla et al. (2014). One report indicated that preparing fresh bull semen by SLC resulted in improved sperm quality in the thawed samples in terms of the proportion of sperm with high mitochondrial membrane potential and with high superoxide production, indicating high metabolic activity (Johannisson et al., 2016). Recent studies with bull spermatozoa in our laboratory have been to develop the reduced volume SLC mentioned earlier (MC Abraham et al.; 2016; Division of Reproduction, Clinical Sciences, Swedish University of Agricultural Sciences, Uppsala, Sweden; unpublished data), and also to use a low density colloid formulation to compare actual fertility of sperm samples in IVF (M Sabés Alsina, JM Morrell, Y Sjunnesson; 2016; Division of Reproduction, Clinical Sciences, Swedish University of Agricultural Sciences, Uppsala, Sweden; unpublished data). One of the problems with using IVF to detect differences between sperm treatments is that the method of preparing the sperm sample can influence the result. Using either swim-up or the normal colloid centrifugation will select motile or good quality spermatozoa, respectively, thus negating the effect of the quality of the original sample. In order to evaluate differences in fertility between treatments a low density colloid can be used just to separate the spermatozoa from the seminal plasma.

\section{Conservation breeding}

Isolated studies of the use of colloids to prepare spermatozoa have been reported in rare or endangered breeds of sheep (Jiménez-Rabadán et al., 2012), donkeys (Ortiz et al., 2015), and bears (AlvarezRodriguez et al., 2016), as well as in cats (Chatdarong et al., 2010), dogs (Dorado et al., 2103), red deer (AnelLopez et al., 2015), llamas (Trassoras et al., 2012) and camels (Malo et al., 2016). In these cases, either the sperm samples were prepared by SLC prior to freezing, to improve cryosurvival, or the most motile spermatozoa were selected from thawed sperm samples to improve their usability. The latter method is very useful when preparing spermatozoa for IVF or ICSI, but may not be so useful for AI particularly if large numbers of .spermatozoa are needed for an insemination dose.

Another article described using a low density colloid to remove somatic cells from an epididymal 
suspension of spermatozoa of the gray wolf, to enable DNA to be extracted (Muñoz-Fuentes et al., 2014). In this case it is important to recover all the sperm cells while separating them from non-sperm cells in the sample. In other cases, epididymal sperm samples may be needed for gamete cryopreservation, in which case it is important to select mature spermatozoa and separate them from cellular debris in the sample. This aspect is particularly relevant for conservation breeding purposes, when it is important to rescue genetic material from rare individuals, which may mean extracting epididymal spermatozoa after the death of the animal. Testicular fragments may also be a source of germplasm for conservation purposes; recent studies with cat testicular tissue showed that SLC could be used to enrich testicular sperm cells from cell suspensions (Chatdarong et al., 2016). The latter authors conclude that SLC could be a useful selection tool for recovering testicular sperm cells from wild cats post mortem for conservation purposes.

One of the challenges with creating gene banks for species conservation is to cryopreserve material in such a manner that it will be usable at a future time. Few checks are carried out to see if frozen spermatozoa retain fertilizing capacity when thawed, partly because of the lack of suitable females or the opportunity to carry out a controlled fertility trial. Many sperm samples are frozen in media containing egg yolk or similar material of animal origin, with the result that it may not be appropriate to use it in the future from the point of view of disease transmission. Although breeding males of domestic species are tested for various virus diseases before freezing their semen, such a luxury is not practical for wild animals. In addition, it is only possible to test for known viruses; there is no means of knowing whether "emerging" diseases have been present in the wild population for some time, with the result that it may be possible to infect or re-infect animal populations by using untested frozen sperm samples.

\section{Removal of pathogens}

As mentioned previously, SLC has been used to separate spermatozoa from bacteria contaminating the ejaculate during semen collection (reviewed by Morrell and Wallgren, 2014). Almost all semen samples are contaminated by bacteria during semen collection and these bacteria tend to multiply due to the ready availability of nutrients supplied by the semen extender. Apart from competing with spermatozoa for nutrients in semen extenders, bacteria may produce metabolic byproducts that are detrimental to spermatozoa, and Gram-negative bacteria produce lipopolysaccharide from their cell walls that is toxic to spermatozoa. High bacterial loads in semen doses can cause a decrease in sperm motility and viablity, and also an increase in agglutination and in the acrosome reaction. Females inseminated with such contaminated semen may return to oestrus after insemination, or there may be high embryonic mortality, endometritis, systemic infection and/or disease, or a reduced litter size in polytocous species.

Therefore, antibiotics are added to semen extenders to control bacterial growth in semen doses for international trade. The antibiotics to be used and the doses are specified in various regulations e.g. European Council Directive 90/429/EEC, Annex C2 (European Union, 2012). However, such a non-therapeutic use of antibiotics is problematic in view of current attempts to reduce antibiotic use. It is now known that antibiotic resistance can develop very quickly and spread to other bacterial species within the same host, or even in different hosts. Therefore, SLC offers an alternative to the use of antibiotics since it can separate spermatozoa from a large proportion of the contaminating bacteria in a sperm sample. Some bacteria are more difficult to remove than others, e.g. those that tend to aggregate or form biofilms, presumably because the density of the "unit" formed is then similar to that of the spermatozoa, or because some bacteria can hook on to spermatozoa and are thus carried through the colloid.

\section{Economics of Single Layer Centrifugation}

The disadvantage of using colloid centrifugation to prepare sperm samples for IVF or ICSI is the extra cost involved. It is very difficult to determine the "value" of embryos produced from particular sires, which makes it impossible to generalise about the economics of using SLC to prepare sperm samples. However, since the sperm samples have to be separated from seminal plasma and/or cryoextender, SLC takes less time than swim-up and has the advantage of selecting spermatozoa with good chromatin integrity. In addition, semen from a male of superior genetic merit is usually used, resulting in embryos of considerable "value". The added advantage of being able to process the semen on only $1 \mathrm{ml}$ of colloid instead of the $4 \mathrm{ml}$ used previously, adds considerably to the merits of SLC as a selection technique. Thus the extra cost involved in purchasing colloid will be more than compensated by the production of more embryos or their enhanced survival and implantation rate. Other factors such as the biosecurity of the semen, reduced antibiotic usage, and the requirements of embryo production for export, must also be considered. Thus, any processing steps that can improve the quality of the semen, and potentially the number of good quality embryos produced, will add value to embryo production.

\section{Concluding remarks}

Sperm selection techniques are needed to prepare spermatozoa for assisted reproduction. Colloid centrifugation, especially Single Layer Centrifugation, can be particularly beneficial since it not only separates the best quality spermatozoa but also separates them from bacteria and viruses that may be present in seminal plasma. The fertility trials that have been carried out to date in a limited number of species indicate that the selected spermatozoa may have enhanced fertilizing capacity compared to unselected controls. If these 
observations also hold for other species, especially rare breeds and endangered species, the technique will be particularly relevant for conservation breeding. Recent developments in reducing the volume of colloid needed to prepare bull spermatozoa for IVF may be particularly advantageous, especially when deciding which sperm preparation technique to adopt.

\section{References}

Abraham MC, Johannisson A, Lopez-Alsina M, Ruete A, Morrell JM. 2016. Comparison of three variants of colloid centrifugation for sperm selection: sperm quality and blastocyst development in vitro. Anim Reprod Sci, 169. doi.org/10.1016/j.anireprosci.2016.03.013.

Alvarez-Rodriguez M, Alvarez M, Anel-Lopez L, Lopez-Ursena E, Manrique P, Borregan S, Morrell JM, de Paz P, Anel L. 2016. Effect of colloid (Androcoll-Bear, Percoll and PureSperm) selection on the freezability of brown bear (Ursus arctos sperm). Theriogenology, 85:1097-1105.

Avery B, Greve T. 1995 Impact of Percoll on bovine spermatozoa used for in vitro insemination. Theriogenology, 44:871-878.

Anel-López L, Martínez-Rodríguez C, Soler AJ, Fernández-Santos MR, Garde JJ, Morrell JM. 2015. Use of Androcoll-S after thawing improves the quality of electroejaculated and epididymal sperm samples from red deer. Anim Reprod Sci, 158:68-74.

Balaban B, Lundin K, Morrell JM, Tjellström H, Urman B, Holmes PV. 2003. An alternative to PVP for slowing sperm prior to ICSI. Hum Reprod, 18:1-3.

Blomqvist G, Persson M, Wallgren M, Wallgren $\mathbf{P}$, Morrell JM. 2011. Removal of virus from boar semen spiked with porcine circovirus type 2. Anim Reprod Sci, 126:108-114.

Cesari A, Kaiser GG, Mucci N, Mutto A, Vincenti A, Fornes MW, Alberio RH. 2006. Integrated morphophysiological assessment of two methods for sperm selection in bovine embryo production in vitro. Theriogenology, 66:1185-1193.

Chatdarong K, Thuwanut P, Morrell JM. 2010 Single-layer centrifugation through colloid selects improved quality of epididymal cat sperm. Theriogenology, 73:1284-1292.

Chatdarong K, Thuwanut P, Morrell JM. 2016. The development of cat testicular sperm cryopreservation protocols: effects of tissue fragments or sperm cell suspensions. Theriogenology, 85:200-206.

Colleoni S, Lagutina I, Rodriguez-Martinez H, Lazzari G, Galli C, Morrell JM. 2011. New techniques for selecting spermatozoa for equine assisted reproduction. J Equine Vet Sci, 31:536-541.

Dorado J, Gálvez MJ, Morrell JM, Alcaráz L, Ortiz I, Acha D, Hidalgo M. 2013. Use of single layer centrifugation with Androcoll-C to enhance sperm quality in frozen-thawed dog semen. Theriogenology, 80:955-962.

European Union. Commission Implementing Regulation (EU) No 176/2012 of 1 March 2012. Amending Annexes B, C and D to Council Directive 90/429/EEC. Available on: http://faolex.fao.org/docs/ pdf/eur110397.pdf.

Goodla L, Morrell JM, Yusnizar Y, Stålhammar H, Johannisson A. 2014. Quality of bull spermatozoa after preparation by single layer centrifugation. J Dairy Sci, 97:2204-2212.

Hoogewijs M, Morrell JM, Van Soom A, Govaere J, Johannisson A, Piepers P, De Schauwer C, de Kruif A, De Vliegher S. 2011. Sperm selection using single layer centrifugation prior to cryopreservation can increase post thaw sperm quality in stallions. Equine Vet $J, 43$ (suppl. 40):35-41.

Hoogewijs M, Piepers S, Govaere J, De Schauwer C, de Kruif A, Morrell JM. 2012. Sperm longevity following pre-freeze sperm selection. $J$ Equine Vet Sci, 32:489.

Jiménez-Rabadán P, Morrell JM, Johannisson A, Ramón M, García-Álvarez O, Maroto-Morales A, Alvaro-García PJ, Pérez-Guzmán MD, FernándezSantos MR, Garde JJ, Soler AJ. 2012. Single layer centrifugation (SLC) improves sperm quality of cryopreserved Blanca-Celtibérica buck semen. Anim Reprod Sci, 136:47-54.

Johannisson A, Morrell JM, Thorén J, Jonsson M, Dalin A-M, Rodriguez-Martinez H. 2009. Colloidal centrifugation with Androcoll-ETM prolongs stallion sperm motility, viability and chromatin integrity. Anim Reprod Sci, 116:119-128.

Johannisson A, Nongbua T, Edman A, Morrell JM. 2016. Effects of single layer centrifugation (SLC) on bull spermatozoa prior to freezing on post-thaw semen characteristics. Theriogenology, 86:140.

Kruse, R, Dutta PC, Morrell JM. 2011. Colloid centrifugation removes seminal plasma and cholesterol from boar spermatozoa. Reprod Fertil Dev, 23:858-865. Lindahl J, Dalin A-M, Stuhtmann G, Morrell JM. 2012. Stallion spermatozoa selected by single layer centrifugation are capable of fertilization after storage for up to $96 \mathrm{~h}$ at $6^{\circ} \mathrm{C}$ prior to artificial insemination. Acta Vet Scand, 54:40-45.

Malo C, Morrell JM, Crichton EG, Pukazhenthi BS, Skidmore JA. 2016. Use of colloid single layer centrifugation for dromedary camel semen: effect of initial dilution and comparison of two colloids on sperm quality parameters. Anim Reprod Sci, 169:123.

Martinez-Alborcia MJ, Morrell JM, Parrilla I, Barranco I, Vázquez JM, Martinez EA, Roca J. 2012. Improvement of boar sperm cryosurvival by using Single-layer colloid centrifugation prior to freezing. Theriogenology, 78:1117-1125.

Matás C, Sansegundo M, Ruiz S, García-Vázquez FA, Gadea J, Romar R, Coy P. 2010. Sperm treatment affects capacitation parameters and penetration ability of ejaculated and epididymal boar spermatozoa. Theriogenology, 74:1327-1340.

Mizuno K, Hoshi K, Huang T. 2002. Fertilization and embryo development in a mouse ICSI model using human and mouse sperm after immobilisation in polyvinlypyrrolidone. Hum Reprod, 17:2350-2355.

Mortimer D. 2000. Sperm preparation methods. $J$ Androl, 21:357-366.

Motoishi M, Goto K, Tomita K, Ookutsu S, Nakanushi Y. 1996. Examination of the safety of 
intracytoplasmic injection procedures by using bovine oocytes. Hum Reprod, 11:618-620.

Morrell JM, Johannisson A, Dalin A-M, RodriguezMartinez, H. 2009. Single layer centrifugation with Androcoll ${ }^{\mathrm{TM}}-\mathrm{E}$ can be scaled-up to allow large volumes of stallion ejaculate to be processed easily. Theriogenology, 72:879-884.

Morrell JM, Rodriguez-Martinez H. 2009. Biomimetic techniques for improving sperm quality in animal breeding: a review. Open Androl J, 1:1-9.

Morrell JM, Rodriguez-Martinez H. 2010. Practical applications of sperm selection technqiues as a tool for improving reproductive efficiency. Vet Med Int. doi:104061/2011/2984767.

Morrell JM, Rodriguez-Martinez H, Johannisson A. 2010. Single Layer Centrifugation of stallion spermatozoa consistently selects the most robust spermatozoa from the rest of the ejaculate in a large sample size: data from 3 breeding seasons. Equine Vet $J, 42: 579-585$.

Morrell JM, van Wienen M, Wallgren, M. 2011. Single layer centrifugation with Androcoll ${ }^{\mathrm{TM}}-\mathrm{P}$ can be scaled-up to process larger volumes of boar semen. ISRN Vet Sci. doi:10.5402/2011/183412

Morrell JM, Wallgren M. 2011. Removal of bacteria from boar ejaculates by single layer centrifugation can reduce the use of antibiotics in semen extenders. Anim Reprod Sci, 123: 64-69.

Morrell JM. 2012. Stallion sperm selection: past, present and future trends. J Equine Vet Sci, 32:436-440.

Morrell JM, Timoney P, Klein C, Shuck K, Campos J, Troedsson M. 2013. Single layer centrifugation reduces equine arteritis virus titer in the semen of shedding stallions. Reprod Domest Anim, 48:604-612.

Morrell JM, Klein C, Lundeheim N, Erol E, Troedsson MHT. 2014a. Removal of bacteria from stallion semen by colloid centrifugation. Anim Reprod Sci, 145:47-53

Morrell JM, Richter J, Martinsson G, Stuhtmann G, Hoogewijs M, Roels K, Dalin A-M. 2014b. Pregnancy rates are higher after artificial insemination with cooled stallion spermatozoa selected by single layer centrifugation than with control semen doses. Theriogenology, 82:1102-1105.

Morrell JM, Rodriguez-Martinez H, Andersson M 2014c. Colloid centrifugation selects normal spermatozoa from polymorphic bull ejaculates: a case study. Reprod Domest Anim, 49:281-284.

Morrell JM, Stuhtmann G, Meurling S, Lundgren A, Winblad C, Macias Garcia B, Johannisson A. 2014d. Sperm yield after single layer centrifugation with Androcoll-E is related to the potential fertility of the original ejaculate Theriogenology, 81:1005-1011.
Morrell JM, Wallgren M. 2014. Alternatives to antibiotics in semen extenders: a review. Pathogens, 3:934-946.

Muñoz-Fuentes V, Linde Forsberg C, Vilà C, Morrell JM. 2014. Single layer centrifugation separates spermatozoa from diploid cells in epididymal samples from grey wolves, Canis lupus (L.) Theriogenology, 82:773-776.

Ortiz I, Dorado J, Morrell JM, Crespo F, Gosalvez J, Gálvez MJ, Acha D, Hidalgo M. 2015. Effect of single layer centrifugation or washing on frozen-thawed donkey semen quality: do they have the same effect regardless of the quality of the sample? Theriogenology, 84:294-300

Oshio S. 1988. Apparent densities of spermatozoa of various mammalian species. Gamete Res, 20:159-164 .

Parrish JJ, Krogenaes A, Susko-Parrish JL. 1995. Effect of bovine sperm separation by either swim-up or Percoll method on success of in vitro fertilization and early embryonic development. Theriogenology, 44:85969

Pertoft H. 2000. Fractionation of cells and subcellular particles with Percoll. J Biochem Biophys Methods, 44:1-30.

Serafini P, Blank W, Tran C, Mansourian M, Tan T, Batzofin J. 1990. Enhanced penetration of zona-free hamster ova by sperm prepared by Nycodenz and Percoll gradient centrifugation. Fertil Steril, 53:551555.

Sjunnesson YC， Morrell JM， González R. 2013. Single layer centrifugation-selected boar spermatozoa are capable of fertilization in vitro. Acta Vet Scand, 55:20-26

Somfai T, Bodo S, Nagy S, Papp AB, Ivancsics J, Baranyai B, Gocza E, Kovacs K. 2002. Effect of swim-up and Percoll treatment on viability and acrosome integrity of frozen-thawed bull spermatozoa. Reprod Domest Anim, 37:285-290.

Suarez S. 2007. Interactions of spermatozoa with the female reproductive tract: inspiration for assisted reproduction. Reprod Fertil Dev, 19:103-110.

Thys M, Vanadele L, Morrell JM, Mestach J, Van Soom A, Hoogewijs M, Rodriguez-Martinez H. 2009. In vitro fertilising capacity of frozen-thawed bull spermatozoa separated by colloidal centrifugation through single-layer or gradients. Reprod Domest Anim, 44:390-394.

Trasorras V, Giuliano S, Chaves G, Neild D, Agüero A, Carretero M, Pinto M, Baca Castex C, Alonso A, Rodríguez D, Morrell J, Miragaya M. 2012. In vitro embryo production in llamas (Lama glama) from in vivo matured oocytes with raw semen processed with Androcoll-E using defined embryo culture media. Reprod Domest Anim, 47: 562-567. 